\title{
Comparison of transthoracic three dimensional echocardiography with magnetic resonance imaging in the assessment of right ventricular volume and mass
}

\author{
M Vogel, M Gutberlet, S Dittrich, N Hosten, P E Lange
}

\begin{abstract}
Objective-Assessment of right ventricular volume and mass with three dimensional echocardiography and comparison with magnetic resonance imaging.

Methods-Measurements of right ventricular volumes performed on three dimensional datasets acquired by transthoracic echocardiography were compared to those obtained from magnetic resonance imaging performed on the same day. Volumes were measured in end systole and end diastole and ejection fraction calculated. Right ventricular mass was assessed in end systole. With both methods, the areas of a $2 \mathrm{~mm}$ thick slice of the ventricle were manually outlined and multiplied by the slice thickness to obtain slice volume. Slice volumes were multiplied by the number of measured slices to obtain the ventricular volume.
\end{abstract}

Patients-16 patients were studied: three with normal hearts, three after surgical repair of coarctation of the aorta, nine following repair of tetralogy of Fallot, and one with Mustard atrial repair of complete transposition of the great arteries.

Results-Correlation between end diastolic volumes measured by both methods was $r=0.95$ with limits of agreement ranging from -3.5 to $12.5 \mathrm{ml}$; correlation for end systolic volumes was $r=0.87$ with limits of agreement between $-4 \cdot 0$ and $16.4 \mathrm{ml}$; correlation for end systolic right ventricular mass was $r=0.81$ with limits of agreement between -7.0 and $20 \cdot 6 \mathrm{~g}$. Interobserver variability ranged from $4 \cdot 3 \%$ (range $0.2 \%$ to $9 \cdot 3 \%$ ) for end diastolic volume to $7 \cdot 6 \%(1 \cdot 8 \%$ to $15 \cdot 4 \%)$ for mass measurements.

Conclusions-With transthoracic three dimensional echocardiography, end diastolic right ventricular volumes can be assessed with acceptable accuracy in normal hearts and those with enlarged right ventricles, whereas the current method of three dimensional echocardiography is less good for end systolic volumes and not satisfactory for right ventricular mass measurements.

(Heart 1997;78:127-130)

Keywords: right ventricular volume; right ventricular mass; three dimensional echocardiography; magnetic resonance imaging
Evaluation of right ventricular function and mass is important in many congenitally malformed hearts, such as with an atrial septal defect, anomalous pulmonary venous drainage, or tetralogy of Fallot, and in lesions where the right ventricle supports the systemic circulation (for example, discordant atrioventricular and ventriculoarterial connections and cases of complete transposition after atrial repair with a Mustard or Senning operation)..$^{1-3}$ Although the exact determination of right ventricular volume and function is important in a clinical setting, ${ }^{1}$ few centres routinely evaluate right ventricular volumes, since simple geometrical formulas cannot be used reliably. ${ }^{4}$ Recently, three dimensional echocardiography has become available ${ }^{56}$ and has been successfully applied for the assessment of left ventricular volumes ${ }^{7}$ and for in vitro measurement of right ventricular volumes. ${ }^{8}$ The purpose of this study was to examine the accuracy of three dimensional echocardiography in the assessment of right ventricular volumes in vivo by comparing the measurements with data obtained by magnetic resonance imaging. ${ }^{4}$

\section{Methods}

ECHOCARDIOGRAPHY EQUIPMENT

A commercially available Vingmed 800 annular array sector scanner (Vingmed, Horten, Norway) was used interfaced with a Tomtec computer (Tomtec, Munich, Germany), which generates three dimensional reconstructions derived from cross sectional images. Either rotational or "fan-like" scanning from an apical or subxiphoid position was used to acquire data suitable for reconstruction. ${ }^{9}$ The multiple sequential cross sectional images are digitally reformatted and stored as a volume element (voxel) on a Pentium $100 \mathrm{MHz}$ personal computer. ${ }^{9}$ Volume quantitation is based on adding multiple slice volumes. ${ }^{8}$ Firstly the longest axis of the right ventricle is defined. Along this axis, perpendicular $2 \mathrm{~mm}$ thick slices are manually outlined to calculate the area of each slice. This is multiplied by the slice thickness to obtain the volume of each slice; the respective volumes are added to obtain the ventricular volume. Measurements were obtained in end systole and end diastole and ejection fraction calculated as end diastolic minus end systolic volume divided by end diastolic volume. Right ventricular mass was evaluated in end systole by measuring epicar- 
Clinical data of patients with right ventricular volume and mass measurements

\begin{tabular}{lcl}
\hline Patient & Age (years) & Diagnosis \\
\hline 1 & 5 & Normal heart \\
2 & $8 \cdot 8$ & Normal heart \\
3 & $9 \cdot 2$ & Repaired aortic coarctation \\
4 & $9 \cdot 3$ & Repaired aortic coarctation \\
5 & $26 \cdot 2$ & Maran syndrome \\
6 & $13 \cdot 7$ & Complete transposition after Mustard operation \\
7 & 21 & Repaired tetralogy of Fallot \\
8 & 5 & Repaired tetralogy of Fallot \\
9 & $6 \cdot 8$ & Repaired tetralogy of Fallot \\
10 & 7 & Repaired tetralogy of Fallot \\
11 & $7 \cdot 2$ & Repaired tetralogy of Fallot \\
12 & $8 \cdot 9$ & Repaired tetralogy of Fallot \\
13 & 9 & Repaired tetralogy of Fallot \\
14 & $9 \cdot 1$ & Repaired tetralogy of Fallot \\
15 & $9 \cdot 8$ & \\
16 & $10 \cdot 7$ &
\end{tabular}

dial volume, from which the endocardial volume was subtracted to obtain the myocardial volume, which was then multiplied by 1.05 (the specific gravity of the heart muscle). The ventricular septum was not considered to be part of the right ventricle for the purpose of the mass measurements.

\section{MAGNETIC RESONANCE IMAGING}

Magnetic resonance images were acquired with a 1.5 Tesla unit (Gyroscan NT15, Philips Medical Systems). A standard body coil was used. Spin echo localising views were performed in the transverse, coronal, and sagittal planes. With this multisection multiphase technique $^{34}$ axial cuts of the right ventricle were obtained. Right ventricular volume and mass measurements were performed in transverse planes, starting at the right ventricular apex up to the bifurcation of the pulmonary artery. ${ }^{4}$ Between 10 and 17 contiguous sections of $5 \mathrm{~mm}$ thickness were sufficient to cover the right ventricle in its entity. The set of multiphase $\mathrm{T} 1$ weighted gradient echo magnetic resonance images was acquired with the shortest heart rate dependent repetition and echo time, a varying field of view of $30 \times 40$ $\mathrm{mm}^{2}$, a flip angle of $20^{\circ}$, and a $128 \times 256$ matrix. Retrograde gating was performed, as conventional cardiac triggering methods do not provide reliable data during the whole cardiac cycle. ${ }^{4}$ Twelve images per cardiac cycle were achieved and the smallest image considered to be the systolic image, while the image with the largest dimension was considered to be the end diastolic image. Calculation of ventricular volumes and muscle mass was performed by summation of ventricular cavity areas (or myocardial areas for mass measurement) multiplied by section thickness. Areas were manually traced, the papillary muscles were included in the right ventricular volume.

\section{PATIENTS}

We examined 16 patients at a mean age of 11 ( 5 to $26 \cdot 2$ ) years. Three had structurally normal hearts and 13 had various congenital heart defects. The clinical data are listed in the table. The nine patients with surgically repaired tetralogy of Fallot had been operated at a mean age of $3(1.0$ to 10.5$)$ years. All had Doppler echocardiographic evidence of mild $(\mathrm{n}=3)$ to moderate $(\mathrm{n}=6)$ pulmonary regurgitation. For this study only patients with good echocardiographic windows and patients in whom the entire right ventricle could be imaged were chosen. Magnetic resonance imaging and three dimensional echocardiography were performed within 24 hours. Sedation was not necessary for either examination in any of the patients studied.

\section{STATISTICAL ANALYSIS}

Results of right ventricular volume measurements were compared with the actual volumes by simple linear regression analysis. The difference between measured and actual volume was expressed in per cent by dividing the difference by the value of the actual volume. Limits for agreement were calculated using the formulas of Bland and Altman. ${ }^{10}$ Interobserver variability of the echocardiographic measurements was assessed by calculating the standard deviation of the differences between measurements of five randomly selected ventricular volumes performed by two independent observers blinded to the results achieved by the other observer.

\section{Results}

We were able to acquire good quality echocardiographic pictures and magnetic resonance images in all patients. Calculation of right ventricular volume in either end diastole or end systole took between 30 and 40 minutes, depending on right ventricular size which determined the number of slices whose area had to be outlined manually. This time was in addition to the acquisition time of three minutes and the five minutes required by the computer to reformat the acquired data, so that a total of 38 to 48 minutes elapsed before a single volume measurement could be obtained, whereas calculation of right ventricular ejection fraction took 70 to 80 minutes. Magnetic resonance imaging took 15 (12 to 19$)$ minutes and the calculation of volumes took 16 (12 to 20) minutes. The limits for agreement between the end diastolic volume measure-

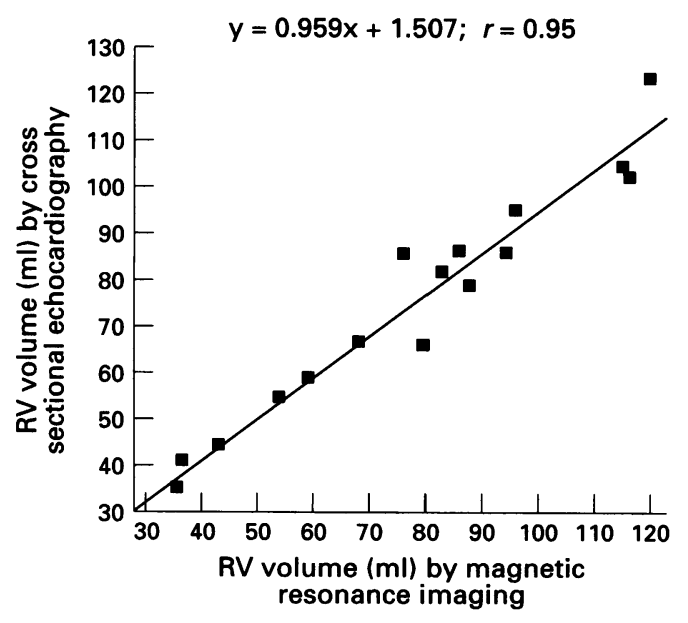

Figure 1 Correlation curve between three dimensional echocardiography and magnetic resonance imaging for end diastolic right ventricular volume. 
Figure 2 Correlation curve between three dimensional echocardiography and magnetic resonance imaging for end systolic right ventricular volume.

Figure 3 Correlation curve between three dimensional echocardiography and magnetic resonance imaging for end systolic right ventricular mass.
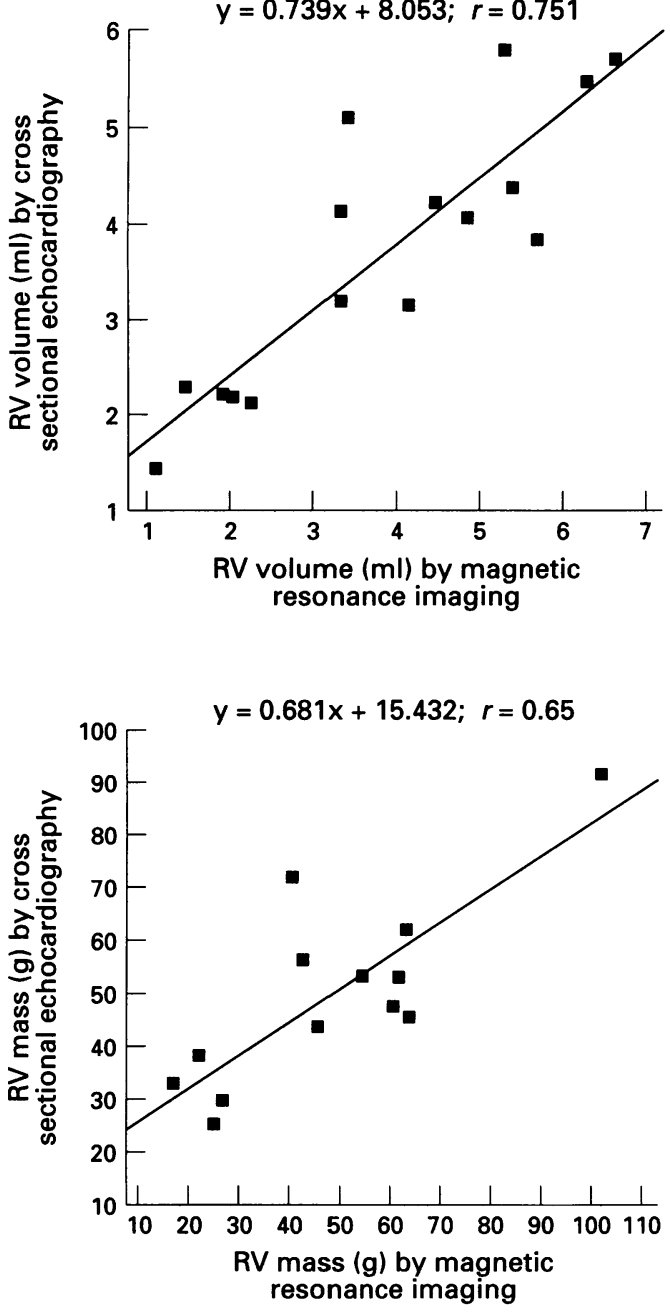

ments by the two methods were -3.5 and $12.5 \mathrm{ml}$, and the correlation curve had an $r$ value of 0.98 . The correlation curves for end diastolic and end systolic right ventricular volume and mass are shown in figs 1-3. Limits for agreement for end systolic volumes were -4.0 to $16.4 \mathrm{ml}$, and the correlation curve had a $r$ value of $0 \cdot 87$. Limits for agreement for ejection fraction were $-3 \cdot 1 \%$ to $16 \cdot 3 \%$ and for right ventricular mass, -7 to $20.6 \mathrm{~g}$; the correlation curve had a $r$ value of 0.81 . Interobserver variability was $4.3 \%(0 \cdot 2 \%$ to $9.3 \%)$ for end diastolic volume, $4.5 \%(0.4 \%$ to $13.6 \%$ ) for end systolic volume, and $7.6 \%$ $(1 \cdot 8 \%$ to $15 \cdot 4 \%)$ for right ventricular mass.

\section{Discussion}

Our data show that end diastolic volumes of normal and enlarged right ventricles can be accurately measured using a commercially available three dimensional echocardiography system, while end systolic volume measurements are less accurate, and mass measurements do not agree well with magnetic resonance imaging.

The echocardiographic method of assessing right ventricular volumes is still cumbersome to use and time consuming and thus cannot yet be applied as a routine clinical tool. It may be improved in the future by using automatic border detection. Magnetic resonance imag- ing-which requires expensive equipment, a long examination time, and uses non-portable hardware-is also of limited clinical use. As volume measurements can be performed with magnetic resonance imaging irrespective of the complex shape of the ventricle, it is considered by some to represent the gold standard of cardiac volume assessment. ${ }^{2-4}$ Differences in agreement between magnetic resonance imaging and three dimensional echocardiography became less good for end systolic volume measurement, probably because right ventricular trabeculations - which may influence identification of endocardial borders - are more pronounced in systole than in diastole. Right ventricular mass measurements showed poor agreement, probably because it was difficult to obtain transthoracic echocardiographic datasets which included the entire epicardium, especially at the apex and the apical free wall. In addition, previous experience with cross sectional echocardiography in the assessment of left ventricular mass showed that the bloodtissue interface is easier to recognise than the myocardial-epicardial interface. ${ }^{11}$ For clinical purposes it is more important, however, that the technique of end diastolic volume measurement by three dimensional echocardiography is reliable in patients with pulmonary incompetence after repair of tetralogy of Fallot $^{12}$ and in transposition of the great arteries after atrial redirection procedures (Mustard or Senning). ${ }^{13}$ These patients may develop important right ventricular dysfunction ${ }^{1213}$ and a reliable preferably non-invasive tool for serial assessment of right ventricular function is required in the long term clinical follow up.

There are some important limitations to this study. For practical purposes we did not assess the influence of the examiner performing the data acquisition. This may be more important than the intraobserver variability in the measurement of the datasets. For assessment of cardiac volumes, meticulous data acquisition is essential as the entire ventricle needs to be imaged and included in the dataset. For this validation study the patients were selected and only those with good transthoracic or subcostal echocardiographic windows were chosen, so that transoesophageal acquisition of data was not needed. However, we are aware that transthoracic acquisition of data suitable for three dimensional reconstruction may not always be possible, especially in adolescents or adults. If transoesophageal data acquisition is required, ${ }^{9}$ echocardiography may become invasive, which limits its clinical use as performing serial studies to monitor right ventricular function may become more difficult.

We conclude that with transthoracic three dimensional echocardiography end diastolic right ventricular volumes can be assessed with acceptable accuracy, whereas the current method of three dimensional echocardiography is less good for end systolic volumes and not satisfactory for right ventricular mass measurements. We submit that one important clinical use of three dimensional echocardiography in the future may be the assessment of right ventricular volume and function. 
1 Oldershaw P. Assessment of right ventricular function and its role in clinical practice. $B r$ Heart $f$ 1992;68: 12-15.

2 Rees S, Warnes C, Underwood R, Firmin D, Klipstein R, Longmore $\mathrm{D}$. Comparison of magnetic resonance imaging with echocardiography and radionuclide angiography in assessing cardiac function and anatomy following Mustard's operation for transposition of the great arteries. Am $\mathcal{F}$ Cardiol 1988;70:1589-95.

3 Helbing WA, Rebergen SA, Maliepaard C, Hansen B, Ottenkamp I, Reiber JHC, et al Quantification of right Ottenkamp J, Reiber JHC, et al. Quantification o children with normal hearts and with congenital heart disease. Am Heart $\mathcal{F}$ 1995;130:828-37.

4 Helbing WA, Bosch HG, Maliepaard C, Rebergen SA, van der Geest RJ, Hansen B, et al. Comparison of echocardiographic methods with magnetic resonance imaging for assessment of right ventricular function in children. $A m \mathcal{F}$ Cardiol 1995;76:589-94

5 Vogel M, Lösch S. Dynamic three-dimensional echocardiography with a computed imaging probe: initial clinica experience with transthoracic application in infants and children with congenital heart defects. Br Heart $\mathcal{f} 1994$ 71:462-7.

6 Vogel M, Ho SY, Lincoln C, Yacoub MH, Anderson RH. Three-dimensional echocardiography can simulate intraoperative visualization of congenitally malformed hearts. Ann Thorac Surg 1995;60:1282-8.

7 Martin RM, Graham MM, Kao R, Bashein G.
Measurement of left ventricular ejection fraction and volumes with three-dimensional reconstructed transesophageal ultrasound scans: comparison to radionuclide and thermal dilution measurements. $\mathcal{f}$ Cardiothorac Anesth 1989;3:260-8.

8 Vogel $M$, White PA, Redington AN. In vitro validation of right ventricular volume measurement by three-dimensional echocardiography. Br Heart $\mathcal{F}$ 1995;74:460-3.

9 Vogel M, Ho SY, Bühlmeyer K, Anderson RH. Assessment of congenital heart defects by dynamic three-dimensional echocardiography: methods of data acquisition and clinical utility. Acta Paediatr Suppl 1995;410:34-9.

10 Bland M, Altman DG. Statistical methods for assessing agreement between two methods of clinical measureagreement between two meth

11 Vogel M, Stern H, Bauer R, Bühlmeyer K. Comparison of magnetic resonance imaging with cross sectional echocardiography in the assessment of left ventricular mass in children without heart disease and in aortic isthmic coarctation. Am $\mathcal{F}$ Cardiol 1992;69:941-4.

12 Redington RN, Oldershaw PJ, Shinebourne EA, Rigby ML. A new technique for the assessment of pulmonary regurgitation and its application to the assessment of right ventricular function before and after repair of tetralogy of Fallot. Br Heart ₹ 1988;60:57-65.

13 Lorenz CH, Walker ES, Graham TP, Powers TA. Right ventricular performance and mass by use of cine MRI late after atrial repair of transposition of the great arteries. Circulation 1995;92(suppl II):II233-9. 